PROCEEDINGS OF THE

AMERICAN MATHEMATICAL SOCIETY

Volume 131, Number 3, Pages 851-857

S 0002-9939(02)06692-3

Article electronically published on July 25, 2002

\title{
DIFFERENTIAL EQUATIONS WITH LIMIT-PERIODIC FORCINGS
}

\author{
ANA I. ALONSO, RAFAEL OBAYA, AND RAFAEL ORTEGA
}

(Communicated by Carmen C. Chicone)

\begin{abstract}
The present paper is concerned with scalar differential equations of first order which are limit periodic in the independent variable. Some tools provided by the theories of exponential dichotomies and periodic differential equations are applied to prove that, in a generic sense, the existence of a bounded solution implies the existence of a limit periodic solution.
\end{abstract}

\section{INTRODUCTION}

A function is limit periodic if it can be obtained as a uniform limit of continuous and periodic functions. The present paper is concerned with the scalar ODE

$$
\dot{x}+g(x)=f(t)
$$

where $f(t)$ is limit periodic. It is well known that if $f$ is periodic, then the existence of a bounded solution in the future implies the existence of a periodic solution in its $\omega$-limit set. This is a consequence of Massera's convergence theorem (see [7]). Johnson [5] gave an example which shows that there are scalar limit periodic differential equations which admit almost automorphic solutions but no limit periodic ones (see also the example of Millionščikov [9, 6]). These examples are not of the precise type (1.1), but they show that an analogous of Massera's theorem does not hold for general limit periodic equations. Almost automorphic solutions are a special class of bounded solutions with some properties of recurrence. They seem to exhibit a sensitive dependence with respect to initial data and small perturbations. In this paper we consider certain Banach spaces composed of limit periodic functions. They are always endowed with the norm of the uniform convergence. Let $X$ denote one of these spaces and let $\mathcal{B}$ be the class of functions $f \in X$ for which (1.1) has a bounded solution. We prove the existence of an open set $\mathcal{G} \subset X$ such that (1.1) admits a limit periodic solution for $f \in \mathcal{G}$ and $\mathcal{G} \subset \mathcal{B} \subset \overline{\mathcal{G}}$. Moreover this limit-periodic solution is hyperbolic and, in consequence, it is uniformly asymptotically stable when $t \rightarrow+\infty$ or $t \rightarrow-\infty$. Section 2 contains a brief summary of the properties of limit periodic functions to prepare Section 3, where our main

Received by the editors October 16, 2001.

2000 Mathematics Subject Classification. Primary 34C11; Secondary 35B15.

Key words and phrases. Limit-periodic equation, hyperbolic solution, almost-automorphic solution.

The first and second authors were partially supported by C.I.C.Y.T. under project PB98-0359 and by Junta de Castilla y León and European community under project VA19/00B. The third author was partially supported by DGES PB98-1294 (Spain).

(C)2002 American Mathematical Society 
results are stated and proved. As a corollary of the main result we show that the Landesman-Lazer conditions lead generically to a limit periodic solution. These conditions are usually employed in the search of periodic or bounded solutions (see [8, [1]).

\section{The MOdule of A Limit PERIOdic FUnCtion}

Let $B C(\mathbb{R})$ denote the Banach space of bounded and continuous functions $\varphi: \mathbb{R} \rightarrow$ $\mathbb{R}$ endowed with the norm of the uniform convergence,

$$
\|\varphi\|=\sup _{t \in \mathbb{R}}|\varphi(t)|,
$$

and let $\mathcal{P} e r \subset B C(\mathbb{R})$ be the class of periodic functions. $\mathcal{P} e r$ is not a vector space because it contains the periodic functions of all periods.

Definition 2.1. A continuous function $f$ is almost periodic if from every sequence of the form $\left\{f\left(t+h_{n}\right)\right\}$, where $h_{n}$ are real numbers, one can extract a subsequence converging uniformly on the real line.

We will denote by $A P(\mathbb{R})$ the space of almost periodic functions. The inclusions

$$
\operatorname{Per} \subset A P(\mathbb{R}) \subset B C(\mathbb{R})
$$

hold and $A P(\mathbb{R})$ becomes a Banach subspace of $B C(\mathbb{R})$. In this section we introduce the definition and main properties of the class of almost periodic functions we are going to deal with.

Definition 2.2. A continuous function $f$ is said to be limit periodic if there exists a sequence of continuous and periodic functions $\left\{f_{n}\right\}$ converging uniformly to $f$.

From the definition, it is clear that limit periodic functions are a subclass of almost periodic functions. Set

$$
L \mathcal{P} e r=\{f \in A P(\mathbb{R}): f \text { is limit periodic }\} .
$$

This is the closure of $\mathcal{P e r}$ in $A P(\mathbb{R})$ and it is not a vector space.

Example 2.3. A typical example of this class of functions is

$$
f(t)=\sum_{n=0}^{\infty} \frac{1}{2^{n}} \sin \left(\frac{t}{2^{n}}\right) .
$$

In this case the functions $f_{n}$ can be chosen as the partial sums of the series and the periods are $2^{n+1} \pi$.

Remark 2.4. Let us recall that two numbers $\alpha$ and $\beta$ in $\mathbb{R}-\{0\}$ are commensurable if they are linearly dependent over $\mathbb{Q}$. In such a case they can be included in a cyclic additive subgroup of $\mathbb{R}$. On the contrary, when $\alpha$ and $\beta$ are not commensurable, the group $\alpha \mathbb{Z}+\beta \mathbb{Z}$ is dense in $\mathbb{R}$.

The next result relates the periods of the periodic approximations of a limit periodic function.

Lemma 2.5. Assume that $f \in L \mathcal{P} e r$ is non-constant and let $\left\{f_{n}\right\}$ be a sequence of functions in $\mathcal{P} e r$, with period $T_{n}>0$ and converging uniformly to $f$. Then there exists $N \geq 0$ such that $T_{n}$ and $T_{m}$ are commensurable if $n, m \geq N$. 
Proof. Let $\tau \in \mathbb{R}$ be such that $f(\tau) \neq f(0)$ and fix $\varepsilon>0$ with $|f(\tau)-f(0)|>4 \varepsilon$. We can find $N$ such that if $n, m \geq N$, then $\left|f_{n}(t)-f_{m}(t)\right| \leq \varepsilon \forall t \in \mathbb{R}$. The same estimate holds for $\left|f(t)-f_{m}(t)\right|$. We are going to prove that $T_{n}$ and $T_{m}(n, m \geq N)$ are commensurable. Otherwise the group $T_{n} \mathbb{Z}+T_{m} \mathbb{Z}$ should be dense and one could find $k_{\nu}, l_{\nu} \in \mathbb{Z}$ with $k_{\nu} T_{n}+l_{\nu} T_{m}$ converging to $\tau$ as $\nu$ goes to $\infty$. For $\nu$ large enough,

$$
\left|f_{m}\left(k_{\nu} T_{n}\right)-f(\tau)\right| \leq 2 \varepsilon
$$

Thus,

$$
\begin{aligned}
|f(\tau)-f(0)| & \leq\left|f(\tau)-f_{m}\left(k_{\nu} T_{n}\right)\right|+\left|f_{m}\left(k_{\nu} T_{n}\right)-f_{n}\left(k_{\nu} T_{n}\right)\right| \\
& +\left|f_{n}(0)-f(0)\right| \leq 4 \varepsilon,
\end{aligned}
$$

and this is not compatible with the definition of $\varepsilon$.

A clear and interesting discussion about the topological structure of the hull of limit periodic functions can be seen in [2]. We shall be more interested in the analytic properties of this class of functions. As limit periodic functions are a subclass of almost periodic functions, we can talk about the exponents and module. We recall these definitions (see [4] for more details). Given $f \in A P(\mathbb{R})$ and $\lambda \in \mathbb{R}$ we define

$$
\widehat{f}(\lambda)=\lim _{T \rightarrow \infty} \frac{1}{T} \int_{0}^{T} f(t) e^{-i \lambda t} d t .
$$

This limit always exists. Define the set of exponents,

$$
\exp (f)=\{\lambda \in \mathbb{R}: \widehat{f}(\lambda) \neq 0\} .
$$

This set is always contable and we define the module of $f, \bmod (f)$, as the smallest subgroup of $\mathbb{R}$ containing $\exp (f)$.

Example 2.6. For the function $f$ defined in Example 2.3 one has

$$
\begin{aligned}
\exp (f) & =\left\{\frac{1}{2^{n}} ; n \geq 0\right\} \cup\left\{-\frac{1}{2^{n}} ; n \geq 0\right\}, \\
\bmod (f) & =\left\{\frac{m}{2^{n}} ; m, n \in \mathbb{Z}\right\} .
\end{aligned}
$$

Proposition 2.7. Let $f$ be a function in $A P(\mathbb{R})$. The following statements are equivalent:

(i) $f$ is limit periodic.

(ii) There exists $\alpha \in \mathbb{R}$ such that $\bmod (f) \subset \alpha \mathbb{Q}$.

Proof. (i) $\Rightarrow$ (ii). We apply Lemma 2.5 and find a sequence $\left\{f_{n}\right\}$ converging uniformly to $f$ and such that all the periods $T_{n}$ are commensurable. Fix $\alpha=\frac{2 \pi}{T_{0}}$. Then $\omega_{n}=\frac{2 \pi}{T_{n}} \in \alpha \mathbb{Q}$ for each $n \geq 1$. Since $\exp \left(f_{n}\right) \subset \omega_{n} \mathbb{Z} \subset \alpha \mathbb{Q}$ we deduce that, for $\lambda \notin \alpha \mathbb{Q}, \widehat{f}_{n}(\lambda)=0$. Thus, passing to the limit, $\widehat{f}(\lambda)=0$. (ii) $\Rightarrow$ (i). The approximation theorem (see Fink [4], page 45) says that we can find a sequence of trigonometric polynomials $p_{n}$ such that $p_{n} \rightarrow f$ uniformly and $\exp \left(p_{n}\right) \subset \alpha \mathbb{Q}$. Since $\exp \left(p_{n}\right)$ is a finite subset of $\alpha \mathbb{Q}$, the $\operatorname{group} \bmod \left(p_{n}\right)$ is cyclic. This implies that $p_{n}$ is periodic and so (i) holds.

Remark 2.8. According to Corduneanu ([3], page 50) the above result was first proved by Bohr in a paper which appeared in 1929. 


\section{A GENERIC RESUlt FOR THE NONLINEAR EQUATION}

Let $G$ be an additive subgroup of $\mathbb{R}$. We define

$$
X_{G}=\{f \in A P(\mathbb{R}): \bmod (f) \subset G\} .
$$

It is easy to check that $X_{G}$ is a closed subspace of $A P(\mathbb{R})$ and so it becomes a Banach space. More than that, $X_{G}$ is a Banach algebra. Actually, given $f, g \in X_{G}$, we find trigonometric polynomials $p_{n}, q_{n}$ with $p_{n} \rightarrow f, q_{n} \rightarrow g$ uniformly and $\exp \left(p_{n}\right), \exp \left(q_{n}\right) \subset G$. It is clear that $\bmod \left(p_{n} q_{n}\right) \subset \bmod \left(p_{n}\right)+\bmod \left(q_{n}\right)$ and so $\bmod \left(p_{n} q_{n}\right) \subset G$. Passing to the limit we deduce that the product $f g$ is in $X_{G}$.

Consider the equation

$$
\dot{x}+g(x)=f(t)
$$

where $g \in C^{2}(\mathbb{R})$ and $f \in X_{G}$. We define

$$
\begin{aligned}
& \mathcal{B}=\left\{f \in X_{G}:(3.1) \text { has a solution in } B C(\mathbb{R})\right\}, \\
& \mathcal{L}=\left\{f \in X_{G}:(3.1) \text { has a solution in } X_{G}\right\} .
\end{aligned}
$$

Clearly $\mathcal{B}$ is a subset of $X_{G}$ which contains $\mathcal{L}$; furthermore $\mathcal{L} \cap \mathcal{P e r}=\mathcal{B} \cap \mathcal{P}$ er (for the proof one could apply Massera's theorem). We can now formulate our main result.

Theorem 3.1. Assume that $G \subset \alpha \mathbb{Q}, \alpha \in \mathbb{R}$, and $\left\{x \in \mathbb{R}: g^{\prime \prime}(x)=0\right\}$ is totally disconnected. Then there exists $\mathcal{G}$, an open subset of $X_{G}$, such that $\mathcal{G} \subset \mathcal{L}$ and $\mathcal{B} \subset \overline{\mathcal{G}}$.

We have divided the proof into a sequence of lemmas. Let us start by recalling the definition of hyperbolic solution of a differential equation. This kind of solution will allow us to get the desired subset $\mathcal{G}$.

Definition 3.2. A solution $x(t)$ in $X_{G}$ of (3.1) is hyperbolic if the linearized equation $\dot{y}+g^{\prime}(x(t)) y=0$ has an exponential dichotomy.

We refer to 4] for the notion of exponential dichotomy. In our case, a scalar equation, this concept can be characterized in terms of the average. Given $f \in$ $A P(\mathbb{R})$ let us denote by $\mathcal{A}[f]$ the average $\mathcal{A}[f]=\lim _{T \rightarrow \infty}(1 / T) \int_{0}^{T} f(t) d t$. The solution $x(t)$ in $X_{G}$ is hyperbolic if and only if $\mathcal{A}\left[g^{\prime} \circ x\right] \neq 0$. Set

$$
\mathcal{G}=\left\{f \in X_{G}: \text { 3.1 has a solution in } X_{G} \text { which is hyperbolic }\right\} \text {. }
$$

Lemma 3.3. Assume that $\varphi \in X_{G}$ is a hyperbolic solution of (3.1). Then there exists $\varepsilon>0$ such that if $h \in X_{G},\|h-f\|<\varepsilon$, then $\dot{x}+g(x)=h(t)$ has a solution in $X_{G}$ which is hyperbolic.

Proof. Let $h \in X_{G}$ and consider the equation $\dot{x}+g(x)=h(t)$. By the change of variables $z=x-\varphi(t)$, we get

$$
\dot{z}+g^{\prime}(\varphi(t)) z=r(t, z)+h(t)-f(t)
$$

where $r(t, z)=-g(z+\varphi(t))+g(\varphi(t))+g^{\prime}(\varphi(t)) z=O\left(z^{2}\right)$ as $z \rightarrow 0$, uniformly in $t \in \mathbb{R}$. Notice that $(\underline{3.2})$ has an almost periodic linear part with $\mathcal{A}\left[g^{\prime} \circ \varphi\right]=$ $\delta \neq 0$. Moreover the function $r$ is almost periodic uniformly on compact sets and $\bmod \left(g^{\prime} \circ \varphi, r\right) \subset G$. To check this inclusion it is convenient to employ the properties of the module described in [4], page 61. It also satisfies a Lipschitz condition of the form

$$
\left|r\left(t, z_{1}\right)-r\left(t, z_{2}\right)\right| \leq L\left(z_{0}\right)\left|z_{1}-z_{2}\right|
$$


with $0 \leq\left|z_{1}\right|,\left|z_{2}\right| \leq z_{0}$ and $\lim _{z_{0} \rightarrow 0} L\left(z_{0}\right)=0$. Under these conditions we can apply Theorem 8.1 of Fink [4] to deduce the existence of a number $\varepsilon_{0}>0$ such that if $\|h-f\|<\varepsilon<\varepsilon_{0}$, then (3.2) has a solution $\psi$ in $X_{G}$ with $\|\psi\| \leq K \varepsilon$. Here $K>0$ is a fixed number which only depends on the linear part. In consequence $\varphi_{h}=\psi+\varphi$ is a solution in $X_{G}$ of $\dot{x}+g(x)=h(t)$. It remains to prove that $\varphi_{h}$ is hyperbolic. Set $l=\|\varphi\|+K \varepsilon_{0}$ and consider the interval $I=[-l, l]$. Then

$$
\mathcal{A}\left[g^{\prime} \circ \varphi_{h}\right]=\lim _{T \rightarrow \infty} \frac{1}{T} \int_{0}^{T}\left(g^{\prime}\left(\varphi_{h}(t)\right)-g^{\prime}(\varphi(t))\right) d t+\mathcal{A}\left[g^{\prime} \circ \varphi\right] \leq\left\|g^{\prime \prime}\right\|_{I} K \varepsilon+\delta,
$$

where $\left\|g^{\prime \prime}\right\|_{I}=\sup _{t \in I}\left|g^{\prime \prime}(t)\right|$. Clearly for every $\varepsilon<\min \left(\varepsilon_{0},|\delta| /\left(K\left\|g^{\prime \prime}\right\|_{I}\right)\right)$ one has $\mathcal{A}\left[g^{\prime} \circ \varphi_{h}\right] \neq 0$, and $\varphi_{h}$ is hyperbolic as claimed.

The previous lemma implies that $\mathcal{G}$ is open. It remains to prove that it is dense in $\mathcal{B}$.

Lemma 3.4. Given $f \in C(\mathbb{R} / T \mathbb{Z}) \cap \mathcal{B}, T>0$, there exists $f_{n} \in C(\mathbb{R} / T \mathbb{Z}) \cap \mathcal{G}$, such that $\left\{f_{n}\right\}$ converges uniformly to $f$.

Proof. Let us assume that $f \in C(\mathbb{R} / T \mathbb{Z}) \cap \mathcal{B}$ is not a constant (otherwise we would approximate it by non-constant functions). Once we know that $\dot{x}+g(x)=f(t)$ has a bounded solution, we can apply Massera's theorem to deduce the existence of a $T$-periodic solution, $\varphi(t)$. Consider the functional

$$
\Phi: C(\mathbb{R} / T \mathbb{Z}) \rightarrow \mathbb{R}, \quad \psi \mapsto \int_{0}^{T} g^{\prime}(\psi(t)) d t .
$$

This is Frechet differentiable and

$$
\Phi^{\prime}(\psi) \delta=\int_{0}^{T} g^{\prime \prime}(\psi(t)) \delta(t) d t
$$

hence the function $\mathbb{R} \rightarrow \mathbb{R}, \varepsilon \mapsto \Phi(\varphi+\varepsilon \delta)$ has derivative

$$
\frac{d}{d \varepsilon} \Phi(\varphi+\varepsilon \delta)_{\left.\right|_{\varepsilon=0}}=\int_{0}^{T} g^{\prime \prime}(\varphi(t)) \delta(t) d t
$$

Since $g^{\prime \prime}(\varphi)$ is not identically zero ( $\varphi$ is not a constant) one can find $\delta \in C^{1}(\mathbb{R} / T \mathbb{Z})$ such that this derivative is not zero. In consequence

$$
\int_{0}^{T} g^{\prime}(\varphi(t)+\varepsilon \delta(t)) d t \neq 0
$$

for small $\varepsilon>0$. Define $f_{\varepsilon}(t)=\dot{\varphi}(t)+\varepsilon \dot{\delta}(t)+g(\varphi(t)+\varepsilon \delta(t))$. It is clear that $f_{\varepsilon}$ belongs to $C(\mathbb{R} / T \mathbb{Z}) \cap \mathcal{G}$ and converges uniformly to $f$.

Lemma 3.5. Let $(t, \lambda) \in \mathbb{R} \times[0,1] \mapsto f_{\lambda}(t) \in \mathbb{R}$ be a continuous function with $f_{\lambda} \in C(\mathbb{R} / T \mathbb{Z}), T>0$, for each $\lambda \in[0,1]$. In addition assume that there are two functions $\psi_{0}(t)$ and $\psi_{1}(t)$ in $B C(\mathbb{R}) \cap C^{1}(\mathbb{R})$ satisfying

$$
\dot{\psi}_{0}(t)+g\left(\psi_{0}(t)\right)>f_{0}(t), \quad \dot{\psi}_{1}(t)+g\left(\psi_{1}(t)\right)<f_{1}(t), \quad t \in \mathbb{R} .
$$

Then there exists $\lambda \in[0,1]$ such that the equation

$$
\dot{x}+g(x)=f_{\lambda}(t)
$$

has a T-periodic solution. 
Proof. The solution of (3.3) with $x(0)=\xi$ will be denoted by $x(t ; \xi, \lambda)$. In some cases these solutions will not be globally defined in $(-\infty,+\infty)$ and, to take care of this possibility, we shall distinguish two cases. Let $C>0$ be a bound of $\left\|f_{\lambda}\right\|$ which is independent of $\lambda$.

Case I. lim $\sup _{|x| \rightarrow \infty}|g(x)|>C$.

Assume for instance that there exists a sequence $\xi_{n} \rightarrow-\infty$ with $g\left(\xi_{n}\right)>C$ (the remaining cases are similar). We choose $N$ large enough so that $\xi_{N}<-\left\|\psi_{1}\right\|$ and use the theory of differential inequalities. For $\lambda=1$ and $\xi$ between $\xi_{N}$ and $\psi_{1}(0)$, the solution $x(t ; \xi, 1)$ is defined in $(-\infty, 0]$ and lies between $\xi_{N}$ and $\psi_{1}(t)$. We can now apply Massera's theorem to conclude that (3.3) has a $T$-periodic solution for $\lambda=1$.

Case II. limsup $\sup _{|x| \rightarrow \infty}|g(x)| \leq C$.

Now all solutions are defined in $(-\infty,+\infty)$. The Poincaré function is $P_{\lambda}(\xi)=$ $x(T ; \xi, \lambda)$. We recall some facts about scalar periodic differential equations (see [10, chapter 2). If for some $\lambda$ the equation (3.3) has no periodic solutions, then either $P_{\lambda}(\xi)>\xi, \forall \xi \in \mathbb{R}$ or $P_{\lambda}(\xi)<\xi, \forall \xi \in \mathbb{R}$. In the first case all the solutions of (3.3) satisfy $\lim _{t \rightarrow+\infty} x(t)=+\infty$ while in the second case one has $\lim _{t \rightarrow+\infty} x(t)=-\infty$. Let us now assume that there are no periodic solutions of (3.3) for $\lambda=0$ and $\lambda=1$. For $\lambda=0$ the function $\psi_{0}$ acts as an upper solution and the solutions below $\psi_{0}$ (for the future) cannot go to $+\infty$. This implies

$$
P_{0}(\xi)<\xi \forall \xi \in \mathbb{R} .
$$

In an analogous way one concludes that

$$
P_{1}(\xi)>\xi \forall \xi \in \mathbb{R} .
$$

The continuity of $P_{\lambda}(\xi)$ implies the existence of $\lambda^{\star} \in(0,1)$ and $\xi^{\star} \in \mathbb{R}$ such that $P_{\lambda^{\star}}\left(\xi^{\star}\right)=\xi^{\star}$. The solution $x\left(t ; \xi^{\star}, \lambda^{\star}\right)$ is $T$-periodic.

We are now in a position to complete the proof of Theorem 3.1 Take $f \in \mathcal{B}$ and let $\varphi(t)$ be a bounded solution of (3.1). Given $\epsilon>0$ one can find $f^{\star} \in \mathcal{P} e r \cap X_{G}$ with $\left\|f-f^{\star}\right\|<\epsilon / 2$. Let $T>0$ be the period of $f^{\star}$. We apply Lemma 3.5 with $f_{\lambda}=f^{\star}+\epsilon\left(\lambda-\frac{1}{2}\right)$ and $\psi_{0}=\psi_{1}=\varphi$. In this way we find a certain $f_{\lambda}$ belonging to $C(\mathbb{R} / T \mathbb{Z}) \cap \mathcal{B}$ and such that $\left\|f_{\lambda}-f\right\|<\epsilon$. From Lemma 3.4 we find $f^{* *} \in \mathcal{P} e r \cap \mathcal{G}$ as close as wished to $f_{\lambda}$. This proves that $\mathcal{B} \subset \overline{\mathcal{G}}$.

Corollary 3.6. In addition to the conditions of Theorem 3.1 assume that $g$ has limits at $\pm \infty$ (finite or infinite) and they are different, say $g(-\infty)<g(+\infty)$. Then there exists $\mathcal{G}^{*}$, open and dense in $X_{G}$, such that (3.1) has a solution in $X_{G}$ if

$$
f \in \mathcal{G}^{*} \quad \text { and } \quad g(-\infty)<\mathcal{A}[f]<g(+\infty) .
$$

Proof. Define $\mathcal{G}^{*}=\mathcal{G} \cup\left(X_{G}-\overline{\mathcal{B}}\right)$ where $\mathcal{G}$ is given by the Theorem. This set is clearly open and dense. Given $f \in X_{G}$ with $g(-\infty)<\mathcal{A}[f]<g(+\infty)$, it is well known that (3.1) has a bounded solution (see for instance [1]). Thus $f$ must belong to $\mathcal{B}$. Now $f \in \mathcal{B} \cap \mathcal{G}^{*}$ implies $f \in \mathcal{G} \subset \mathcal{L}$ and so there exists a limit periodic solution. 


\section{REFERENCES}

[1] S. Ahmad, A nonstandard resonance problem for ordinary differential equations, Trans. Am. Math. Soc. 323 (1991), 857-875. MR 91e:34046

[2] H. Bell \& K. Meyer, Limit periodic functions, adding machines and solenoids, J. Dynamics and Diff. Eqs. 7 (1995), 409-422. MR 96m:58130

[3] C. Corduneanu, Almost periodic functions, J. Wiley 1968. MR 58:2006

[4] A.M. Fink, Almost periodic differential equations. Lecture Notes in Mathematics 377, Springer-Verlag, Berlin, Heidelberg, New York 1974. MR 57:792

[5] R.A. Johnson, A linear almost periodic equation with an almost automorphic solution, Proc. Amer. Math. Soc. 82 (1981), 199-205. MR 82i:34044a

[6] R.A. Johnson, On almost-periodic linear differential systems of Millionščikov and Vinograd, J. Math. Anal. App. 85 (1982), 452-460. MR 84h:34068

[7] J.L. Massera, The existence of periodic solutions of systems of differential equations, Duke Math. J. 17 (1950), 457-475. MR 12:705g

[8] J. Mawhin, Landesman-Lazer conditions for boundary value problems: a nonlinear version of resonance, Boletín de la Sociedad Española de Matemática Aplicada 16 (2000), 45-65.

[9] V.M. Millionščikov, Proof of the existence of irregular systems of linear differential equations with almost periodic coefficients, Differential Equations 4 (1968), 203-205. MR 37:5478

[10] V.A. Pliss, Nonlocal problems of the theory of oscillations. Academic Press, New York 1966. MR 33:4391

E.T.S.I.I. Departamento de Matemática Aplicada a la Ingeniería, Paseo del Cauce S/N, 47011 Universidad de VAlladolid, Spain

E-mail address: anaalo@wmatem.eis.uva.es

E.T.S.I.I. Departamento de Matemática Aplicada a la Ingeniería, Paseo del Cauce S/n, 47011 Universidad de VAlladolid, Spain

E-mail address: rafoba@wmatem.eis.uva.es

Departamento de Matemática Aplicada, Facultad de Ciencias, Universidad de Granada, 18071 Granada, Spain

E-mail address: rortega@ugr.es 